\title{
FoxH1 represses miR-430 during early embryonic development of zebrafish via non-canonical regulation
}

\author{
Patrick Fischer ${ }^{1}$, Hao Chen ${ }^{1}$, Frederic Pacho ${ }^{1}$, Dietmar Rieder ${ }^{2}$, Robin A. Kimmel ${ }^{1}$ and Dirk Meyer ${ }^{1 *}$ (D)
}

\begin{abstract}
Background: FoxH1 is a forkhead transcription factor with conserved key functions in vertebrate mesoderm induction and left-right patterning downstream of the TGF-beta/Nodal signaling pathway. Binding of the forkhead domain (FHD) of FoxH1 to a highly conserved proximal sequence motif was shown to regulate target gene expression.

Results: We identify the conserved microRNA-430 family (miR-430) as a novel target of FoxH1. miR-430 levels are increased in foxH1 mutants, resulting in a reduced expression of transcripts that are targeted by miR-430 for degradation. To determine the underlying mechanism of miR-430 repression, we performed chromatin immunoprecipitation studies and overexpression experiments with mutant as well as constitutive active and repressive forms of FoxH1. Our studies reveal a molecular interaction of FoxH1 with miR-430 loci independent of the FHD. Furthermore, we show that previously described mutant forms of FoxH1 that disrupt DNA binding or that lack the C-terminal Smad Interaction Domain (SID) dominantly interfere with miR-430 repression, but not with the regulation of previously described FoxH1 targets.
\end{abstract}

Conclusions: We were able to identify the distinct roles of protein domains of FoxH1 in the regulation process of miR430. We provide evidence that the indirect repression of miR-430 loci depends on the connection to a distal repressive chromosome environment via a non-canonical mode. The widespread distribution of such non-canonical binding sites of FoxH1, found not only in our study, argues against a function restricted to regulating miR-430 and for a more global role of FoxH1 in chromatin folding.

Keywords: Gastrulation, Nodal signaling, FoxH1, miR-430, MZT, Chromatin folding

\section{Background}

Gastrulation and hence formation of the three germ layers endoderm, mesoderm, and ectoderm are a key step in development from single-cell to multicellular organism. Among other pathways, dose-dependent signaling by the TGF-beta factor Nodal is central for germ layer induction and patterning. Throughout the vertebrate phylogeny, the loss of Nodal signaling leads to loss of endodermal and mesodermal cell fate [1-3]. A central step in Nodal signaling is the ligand-induced phosphorylation and subsequent nuclear translocation of Smad2. Within the nucleus, Smad2 forms a complex with Smad4 and interacts with different transcription factors, each

\footnotetext{
*Correspondence: dirk.meyer@uibk.ac.at

'Institute of Molecular Biology/CMBI, University of Innsbruck, Technikerstrasse 25, 6020 Innsbruck, Austria

Full list of author information is available at the end of the article
}

targeting the SMADs to a different set of target genes ([4-6], reviewed in [7]). The transcription factor FoxH1 was the first SMAD-interacting protein to be identified. Genetic studies established conserved requirements for FoxH1 in mediating Nodal activities related to mesoderm induction and left-right patterning [8-12]. Consistent with the genetic requirements, molecular studies identified several mesoderm-related transcription factors (e.g., tbxta, tbx6, foxA3, pitx2) and signaling molecules (e.g., FGF8, FGF3, Wnt11) as conserved FoxH1 targets. FoxH1 is further required for modulating Nodal signaling intensity, range, and duration by directly regulating Nodal and Lefty encoding genes, with the latter being a Nodal antagonist $[8,13-16]$.

FoxH1 binds chromatin via the conserved canonical (CAN) consensus motif AATMCACA. CAN binding sites critical for mediating Nodal signals are further characterized 
by close-by SMAD binding sites (SBS) [8, 16-19]. Currently, it is not entirely clear whether FoxH1 can bind chromatin without the Smad2/3 interaction. Recent work in pluripotent mouse P19 cells showed that binding of FoxH1 to SBS-associated CAN enhancers is strictly Smad2 dependent [20]. However, studies in human embryonic stem cells and very recent findings in frog embryos showed that FoxH1 can be pre-positioned at specific enhancers and that the subsequent interaction with activated Smad2 can induce a release or a switch from repression to activation of associated genes $[9,21]$.

The FoxH1 protein has two functionally well-defined domains, an N-terminal forkhead domain (FHD) for DNA-binding and a C-terminal domain required for interaction with activated SMADs (SID, Smad interaction domain). An additional medially positioned conserved EH1 motif was recently shown to mediate Nodal-independent transcriptional repression via interaction with Groucho/ TLE factors [22, 23]. Genetic analyses in zebrafish revealed slightly different phenotypes in foxH1 mutant embryos lacking either a functional FHD (sur/schmalspur) or the SID (mid/midway). In maternal and zygotic (MZ) mutant embryos, both types of foxH1 mutations cause defects in axial mesoderm formation, a loss of ventral neural fates, ventral body curvature, and synophthalmia. However, while axial mesoderm is partially disrupted in MZsur $\left(=\right.$ foxH $\left.1^{m 786}\right)$ mutants $[10,24]$, it is missing in MZmid $\left(=f o x H 1^{P r 1}\right)$ mutants and the embryos display additional defects in somite patterning. Since MZmid mutants can be rescued to an MZsur like phenotype by injection of sur mutant foxH $1^{m 786}$ mRNA, it was suggested that DNA-binding defects in sur mutants might be partially compensated by the interaction of the FoxH $1^{m 786} /$ SMAD complex with other DNA-binding proteins [11]. Consistent with this option, canonical FoxH1 binding sites are frequently found in association with binding sites for other Smad2-interacting transcription factors such as Eomes and Mixl1 [12].

Interestingly, previous ChIP analyses revealed that the majority of FoxH1 peaks in fact lack CAN FoxH1 consensus motifs $[12,25]$. The molecular nature and functional relevance of these peaks, termed non-canonical (NC) peaks, have not been addressed so far. In this study, we identified the miR-430 loci as targets for NC interaction with FoxH1 and we show that this interaction is relevant for FoxH1 dependent miR-430 repression.

In zebrafish, 415 different miRNAs in 44 families have been found so far ([26], overview in [27]). Among those, three isoforms of miR-430 could be distinguished (a, b, $c)$, which differ in their central and terminal nucleotide sequence, but are homologous in the $3^{\prime}$ region and target recognition site at the $5^{\prime}$ end ([28-30], overview in [27]). The majority of miR-430s are transcribed from a large cluster on chromosome 4 that contains more than
50 copies of miR-430 [31]. Like most vertebrate miRNAs, $m i R-430$ are also transcribed as long primary transcripts (pri-miR-430). These transcripts undergo a maturation process: cleavage into hairpin-structured precursor miRNA (pre-miR-430) by a protein complex of Drosha and its cofactor DGCR8, transport into cytoplasm, generation of a 22-nucleotide long imperfect RNA duplex by Dicer and cofactors, and loading into the RISC (RNA-induced silencing complex) ([31]; overview in [27]). miR-430 family members are highly expressed at the onset of gastrulation ( $5 \mathrm{~h}$ post fertilization, 5hpf) and stay expressed during gastrulation and somitogenesis [32-36]. The early embryonic expression of $m i R-430$ is required for clearance of maternal RNAs at the time when zygotic expression starts (midblastula transition; Additional file 1: Figure S1 [30, 37]). To this end, miR430 targets hundreds of transcripts for deadenylation and degradation [34]. Furthermore, miR-430 is involved in fine tuning and regulation of Nodal signaling, namely by targeting mRNA encoding the Nodal agonist nodal-related 1 (ndr1; squint) and the antagonist lefty2 [36, 38, 39].

Here we show that NC-binding of FoxH1 at miR-430 loci correlates with a downregulated expression of all three miR-430 subtypes and with a downregulation of miR-430-targets in the MZsur mutant. We further show that the FHD and the SID-containing C-terminus of FoxH1 are both required for miR-430 repression and that mutant forms lacking function of one of these domains dominantly interfere with this activity. Our data demonstrate a novel physiologically relevant requirement for FoxH1 in miR-430 regulation, and they provide a first insight into the mechanism underlying noncanonical gene regulation by FoxH1. The results imply a hypothetical model of indirect FoxH1 activity on miR430 loci that includes chromatin folding effects.

\section{Results \\ ChIP-seq and microarray revealed non-canonical FoxH1 regulation of $m i R-430$}

To gain insight into the function of FoxH1 during early embryonic development, we performed a combination of expression and ChIP-seq analysis on 6hpf epiboly-stage zebrafish embryos. The ChIP analyses [40] revealed $8,342,137$ high-quality reads that could be mapped to more than 16,000 peaks $\left(p<\mathrm{e}^{-4}\right)$ in the zebrafish genome ( $\mathrm{Zv} 9 /$ danRer7). De novo motif prediction algorithms from two different toolsets (MEME-ChIP and RSAT) [41-43] confirmed the presence of the wellestablished FoxH1 consensus motif in about 14\% (2421; Additional file 2: FoxH1-peaks and Annotation-50 k + $20 \mathrm{k}$ ) of these peaks [8,16-19]. Correlation analysis of FoxH1 peaks with published Smad2 binding regions (SBRs) [44] further confirmed overlap between SBRs and FoxH1 peaks containing the consensus motif (termed CAN-peaks) but not with peaks lacking the motif 
(termed NC-peaks) (Fig. 1a; Additional file 3: SBR to FoxH1 peaks).

To correlate chromatin binding with requirements for FoxH1-dependent gene regulation, we performed microarray-based transcriptome analyses. Comparison of expression data from 6hpf epiboly-stage wild-type (biological duplicates) embryos with that of MZsur mutants (biological triplicates) revealed a total of 1575 genes with at least twofold changes in expression (Fig. 1b; Additional file 4: Microarray vs. ChIP).

Consistent with the expected primary role of FoxH1 in Nodal signaling, we also find that the majority of these genes $(76 \%)$ show a similarly changed expression in Nodal signaling-deficient MZoep mutants (Fig. 1c; Additional file 4: Microarray vs. ChIP). To identify potentially direct FoxH1 targets, all regulated genes were analyzed for the presence of FoxH1 peaks within $50 \mathrm{~kb}$ upstream and $20 \mathrm{~kb}$ downstream of the transcriptional start site (TSS). FoxH1 peaks were found for 551 of the genes with twofold changed regulation in MZsur mutants (Fig. 1b; Additional file 4: Microarray vs. ChIP). From those, 127 genes were associated with Smad2/FoxH1 co-binding, which included most of the previously reported Nodal/FoxH1-activated genes (ndr1, lft2, pitx2, flh, foxa3, and lhxla) [3]. Surprisingly, the majority of FoxH1-regulated genes associated with NC-peaks. As FoxH1 has been mainly associated with gene-activating functions, we expected direct targets to be mainly downregulated in the mutants. However, similar numbers of down- and upregulated peakassociated genes in MZsur and MZoep mutants suggested that CAN- and NC-FoxH1 peaks both contributed to Nodal/FoxH1-dependent gene activation and repression (Fig. 1c).

Among NC-peaks, we noted several prominent peaks within the miR-430 "a, b, c" repeat cluster (Additional file 2: FoxH1-peaks and Annotation-50 k+20 k; Fig. 2a [45]). To determine potential requirements of FoxH1 in $m i R-430$ regulation, we analyzed expression of mature miR-430 isoforms (a, b, c) by using a poly-A tailing approach [47]. Consistent with a general role of FoxH1 in $m i R-430$ repression, all three $m i R-430$ isoforms were significantly increased in gastrula stage MZsur mutant embryos (Fig. 2b; Additional file 5: Individual qPCR values). Increased miR-430 transcription in MZsur as compared to wild type was further confirmed by direct RT-qPCR analyses of selected subsets of pri-miR-430 transcripts for the three isoforms (Fig. 2c; Additional file 5: Individual qPCR values).

To confirm that the repressive effect is specific to FoxH1, we further tested whether injection of foxH1 ${ }^{\text {WT }}$ mRNA into MZsur embryos would lower pri-miR-430 levels. Consistent with a specific function, the injected foxH1 ${ }^{W T}$ mRNA reduced pri-miR-430 levels (Fig. 2c; Additional file 5: Individual qPCR values).
Next, we asked whether miR-430 regulation by FoxH1 is Nodal signaling-dependent. Consistent with a role of Nodal signaling in miR-430 repression 7hpf MZoep mutants (Fig. 2d; Additional file 5: Individual qPCR values) show increased pri-miR-430 transcript levels. However, this increase was less prominent as compared to MZsur mutants. As a control, we verified that the canonical Nodal target goosecoid (gsc) is reduced in MZoep and MZsur mutants (Fig. 2e; Additional file 5: Individual qPCR values). Together, these data reveal a novel requirement for FoxH1 in pri-miR-430 repression, and they suggest that the sur mutation has Nodal signalingindependent effects on miR-430 regulation.

\section{Mutations in the FHD and SID of FoxH1 dominantly interfere with miR-430 regulation}

The lack of canonical binding motifs within the miR-430 associated peaks suggests that FoxH1 is not directly interacting with these sites. To further test this, we performed RNA injections with FHD-containing constructs (Additional file 6: Overview of FoxH1 constructs) previously shown to cause robust activation (FHD-VP16) or repression (FHD-EN) on CAN targets (Fig. 3a; Additional file 5: Individual qPCR values) [10, 11]. Analysis of selected CAN-FoxH1 targets in injected embryos showed the expected up- and downregulation by injections of $F H D-V P 16$ and $F H D-E N$, respectively, and virtually no effect following injection of a FHD-GFP control construct (Additional file 7: Figure S2). In contrast, and consistent with an indirect regulatory effect, all three types of injections (FHD-VP16, FHD-EN and FHD-GFP) caused increased pri-miR-430 levels (Fig. 3a; Additional file 5: Individual qPCR values) with FHD-GFP showing the strongest activity not only in wild type but also in MZsur mutants.

The data suggest that the common FHD-containing $\mathrm{N}$-terminus of FoxH1 in these constructs interferes with pri-miR-430 repression in a dominant negative manner. To clarify which parts of the proteins mediate the dominant negative activities, we tested additional GFP fusion constructs (Fig. 3b; Additional file 5: Individual qPCR values, see also Additional file 6: Overview of FoxH1 constructs). In particular, we addressed functions of the conserved C-terminal region that includes the EH1 motif and of the non-conserved $\mathrm{N}$-terminal part. We found that the 88 most $\mathrm{N}$-terminal amino acids of FoxH1 ( $5^{\prime}$-Foxh1) were not sufficient to influence primiR-430 levels and that the removal of the EH1 motif $\left(F H D^{\Delta \mathrm{EH} 1}-G F P\right)$ did not prevent interference with pri$m i R-430$ repression. These data suggest that the FHD, while not directly interacting with the $m i R-430$ loci, is critical for miR-430 regulation. To further test if this critical activity depends on the DNA-binding properties of the FHD, we injected a $s u r^{768}$ mutant variant of FHD- 


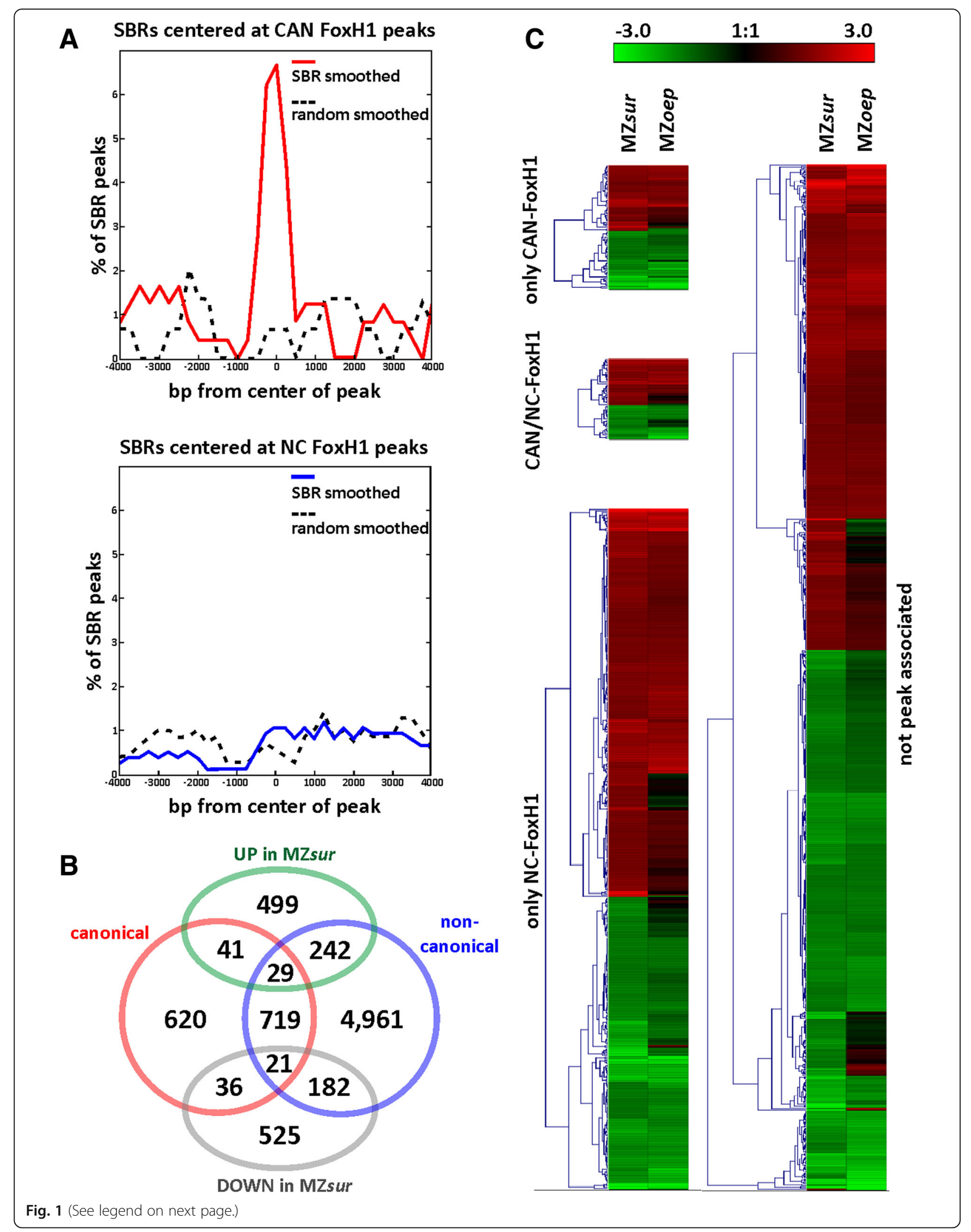


(See figure on previous page.)

Fig. 1 FoxH1 is associated with canonical (CAN) and non-canonical (NC) target sites. a FoxH1 CAN-peaks but not NC-peaks co-localize with SBRs. b Indication of the numbers of genes with more than twofold up (UP) or down (DOWN) regulation in MZsur mutants associated with CAN, NC, or $\mathrm{CAN}+\mathrm{NC}$ peaks of FoxH1. c Heatmap comparison of all FoxH1 peak-associated (left panels) and not FoxH1 associated (right panel) genes with more than twofold changed expression in MZsur (left-side) as compared to wild-type embryos. Note that most genes show very similar changes of expression in MZoep mutants (see right-side for comparison)

GFP (termed FHD ${ }^{\text {m768 }}$-GFP; Additional file 6: Overview of FoxH1 constructs). The single amino acid exchange in FoxH $1^{\mathrm{m} 768}$ has previously been shown to specifically prevent binding to FoxH1-consensus motifs [10]. Since FHD $^{\mathrm{m} 768}$-GFP had no major effect on the regulation of pri-miR-430 (Fig. 3b; Additional file 5: Individual qPCR values), this suggests that CAN-DNA interaction is important for the dominant negative function of FHDGFP.

The data imply that SID-depleted fusion proteins with a wild-type FHD bind to an undefined CAN binding site and thereby prevent repressive interactions of this CANsite with the $m i R-430$ loci. This further suggests that the SID-containing C-terminus of FoxH1 might be required for mediating contact with the $m i R-430$ loci. In this case, overexpression of the FoxH1 ${ }^{m 768}$ protein, with an intact SID but defective FHD, should also interfere with pri-miR430 repression by occupying the $m i R-430$ loci-specific contact site. Consistent with this notion, injection of foxH $1^{m 768}$ mRNA caused twofold increased expression levels of primiR-430 in wild type and surprisingly also in MZsur embryos (Fig. 3c; Additional file 5: Individual qPCR values). To directly address whether a FHD-depleted FoxH1 protein is able to interact with the miR-430 loci, we tested a fusion construct between the C-terminal part of FoxH1 and the EN-repressor domain (termed SID-EN; Additional file 6: Overview of FoxH1 constructs). In contrast to foxH1 $1^{m 768}$ that caused increased pri-miR-430 levels, the injection of $S I D-E N$ resulted in strongly reduced pri-miR-430 levels (Fig. 3d; Additional file 5: Individual qPCR values). Since SID-EN lacks known DNA-binding motifs, we reasoned that its interaction with the miR-430 loci might be mediated through interaction with SMAD2/3 proteins. However, downregulation of pri-miR-430 by SID-EN was also seen in MZoep mutant embryos (Fig. 3d; Additional file 5: Individual qPCR values) which lack phosphorylated and thereby nuclear SMAD proteins. These results suggest a SMAD-independent interaction of the SID with the $m i R-430$ loci.

Together, these data suggest that FHD and the SIDcontaining $\mathrm{C}$-terminus of FoxH1 have unique functions in mediating repressive contact between distal chromatin structures and that injection of FoxH1 proteins lacking one of these domains interfere with this activity in a dominant negative manner.

\section{Injection of FHD-GFP increased severity of MZsur mutant phenotype}

In previous studies, the injection of fox $H 1^{m 768}$ mRNA into MZmid mutants was found to convert the more severe MZmid phenotype into the weaker MZsur phenotype. It was therefore suggested that FoxH $1^{p r 1}$ might be inactive while FoxH $1^{\mathrm{m} 768}$ retains residual FoxH1 activities via the intact SID [11]. Our studies show that both types of mutations interfere with $m i R-430$ regulation. Since the loss of the SID in FHD-GFP, resembling Fox $\mathrm{H}^{p r 1}$, has stronger effects on $m i R-430$ regulation as compared to FoxH $1^{\mathrm{m} 768}$, we reasoned that the stronger phenotype of MZmid could be associated with the stronger dominant-negative effects of the SID-truncated protein. Consistent with this notion, we found that injection of FHD-GFP into MZsur mutants strongly increased the expressivity of the mutant phenotype. WISH analyses for the axial marker col2a1a in $24 \mathrm{hpf}$ embryos confirmed a major effect of FHD-GFP on notochord formation in MZsur mutants (Fig. 4a-d). MZsur mutants lack the floor plate and hypochord and show some variable notochord defects in the tail and anterior trunk [11]. Still, these embryos showed nearly continuous col2a1a staining in the trunk notochord, while more than $60 \%$ of FHD-GFP injected MZsur embryos displayed only patchy col2a1a signals. Analyses of foxa2 expression in gastrula stage embryos indicated that FHD-GFP prevents formation of early axial mesoderm in MZsur (Fig. 4e-h). We found that the number of foxa2-positive axial cells was strongly reduced in FHD-GFP injected MZsur as compared to control and un-injected MZsur embryos. Notably, FHD-GFP also caused broadened axial signal in about $50 \%$ of the injected control embryos, possibly indicating defects in gastrulation movement. The foxa2 stains also suggested that FHD-GFP has mild effects on endoderm formation (arrowheads in Fig. 4e-h). Further analyses of the endoderm marker sox 17 confirmed a slightly reduced amount of endoderm cells, and in addition, they revealed strongly reduced number of forerunner cells in FHD-GFP injected embryos (Fig. 4m-t; Additional file 8: Statistical analysis of forerunner cells).

As the severity of foxH1 mutant phenotype appears to correlate with the level of $m i R-430$ upregulation, we next tested whether an injection of $m i R-430$ blocking dremiR-430 morpholinos (MOs) [36] is able to attenuate 


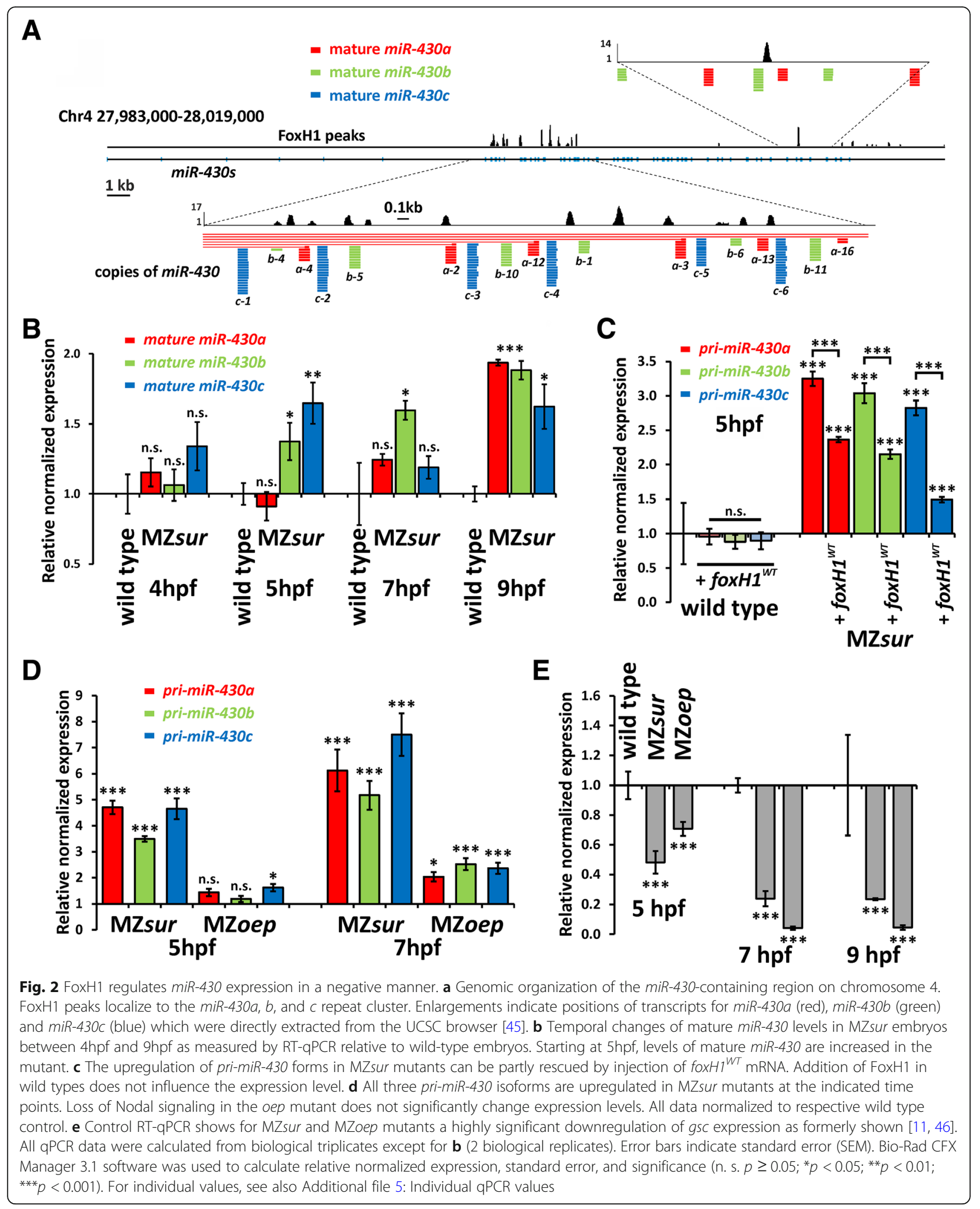



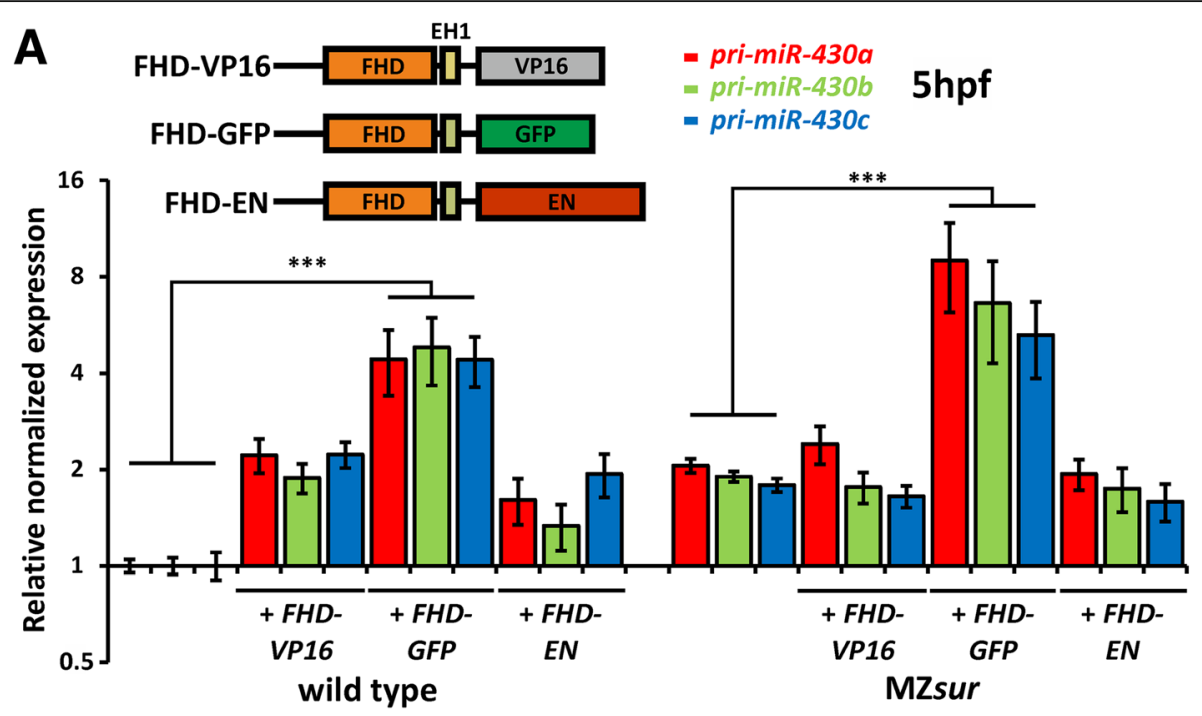

B 5'FoxH1 $\stackrel{1-8}{-8}$

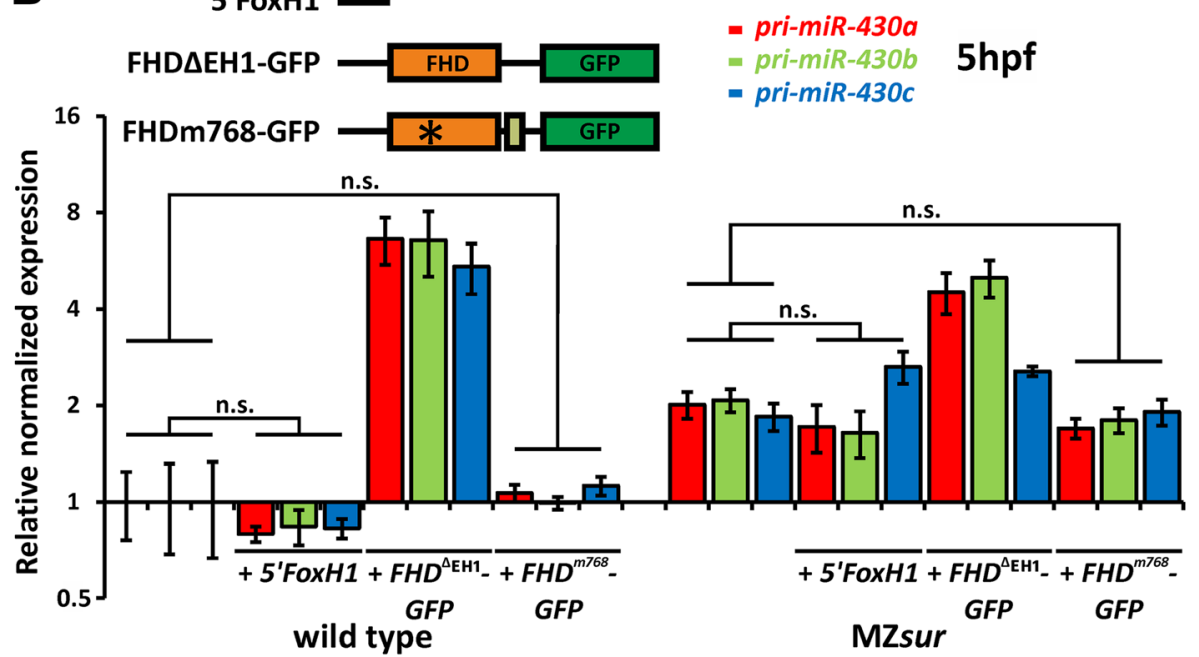

C
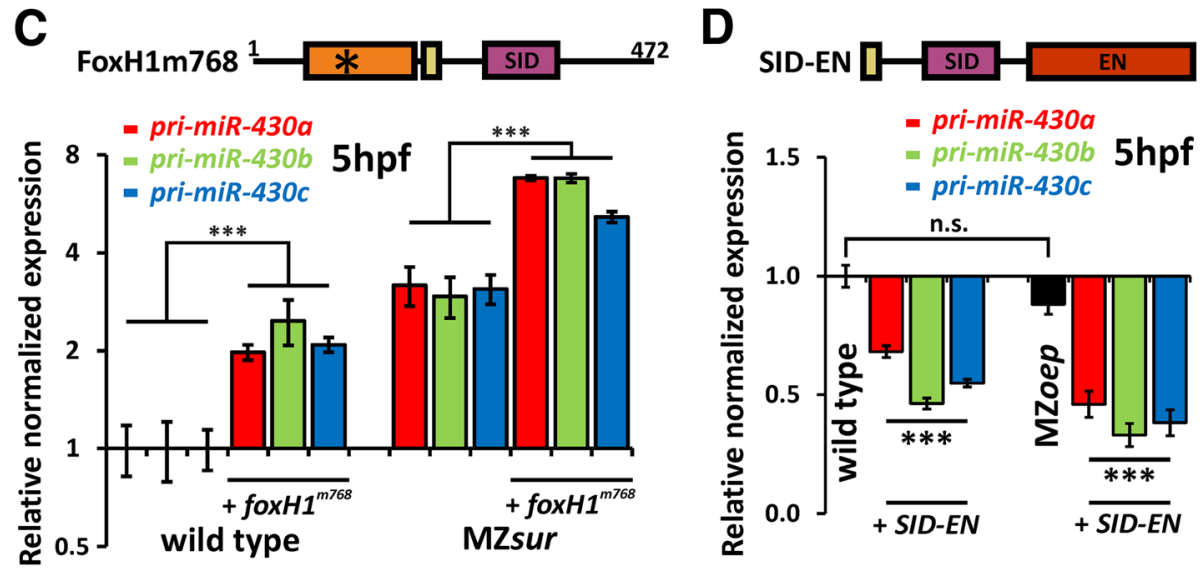

Fig. 3 (See legend on next page.) 
(See figure on previous page.)

Fig. 3 Mutated FoxH1 proteins for SID or FHD reveal dominant-negative effects on pri-miR-430 at 5hpf. a The FHD fused to VP16, EN, or GFP leads to enhanced upregulation in wild-type and MZsur mutants. Note highest upregulation for FHD-GFP constructs. b Sequences $5^{\prime}$ and $3^{\prime}$ to the FHD do not influence expression levels. A sur-mutated form of FHD fused to GFP does also not interfere with the expression level. c Addition of mutant proteins without DNA binding ability enhances the upregulation in wild type and MZsur mutant embryos. $\mathbf{d}$ Injection of SID-EN in both wild type and MZoep mutant embryos resulted in the downregulation of pri-miR-430 expression. The full-length protein consists of 472 aa. FHD (orange) is wild type or mutated to sur allele $\left(^{*}\right)$. SID (purple) can be wild type or replaced by VP16 (gray), GFP (green), or EN (brown). Olivecolored box following the FHD represents the EH1 domain which was included in earlier VP16, GFP, and EN constructs [10]. Error bars indicate standard error (SEM). All experiments were performed as biological triplicates of $5 \mathrm{hpf}$ embryos injected with the indicated mRNA at 1-2-cell stage. Relative normalized expression, standard error, and significance were calculated with the Bio-Rad CFX Manager 3.1 software (n. s. $p \geq 0.05$; $\left.{ }^{* * *} p<0.001\right)$. For individual values, see also Additional file 5: Individual qPCR values

the phenotype of MZsur and FHD-GFP injected MZsur (Fig. 4i-l). However, the morpholino injection had the opposite effect and caused strongly reduced and even absent axial foxa2 signals in MZsur and FHD-GFP injected MZsur, respectively (Fig. 4k, l).

FoxH1 regulation of miR-430 prevents maternal clearance Finally, we asked whether regulation of $m i R-430$ by FoxH1 is functionally relevant in the developing embryo. Based on previous studies, we expected that the increased miR-430 levels in MZsur mutants or RNAinjected embryos would have effects on maternal transcript clearance [34, 47]. To determine functions of FoxH1 in maternal transcript clearance, we analyzed expression of two maternally deposited miR-430 targets, namely $c d 82 b[48,49]$ and jade1 $[50,51]$. Expression analyses of $4-5 \mathrm{hpf}$ embryos by RT-qPCR (Fig. 5a, b; Additional file 5: Individual $\mathrm{qPCR}$ values) and WISH (Fig. 5c-f) show that transcripts levels of $c d 82 b$ and jade1 are significantly lower in MZsur mutants as compared to wild-type embryos and that this phenotype is reduced by injection of foxH $1^{W T}$ mRNA. To exclude a possible direct regulation of these genes by FoxH1 or by canonical FoxH1 targets, we also analyzed expression in FHD-VP16 injected embryos. The similar $c d 82 b$ and jade1 levels in un-injected and FHD-VP16 injected MZsur embryos emphasize the indirect connection between FoxH1 and $c d 82 b / j a d e 1$ and are consistent with the important intermediary role of pri-mir-430 upregulation, as seen following injection of FHD-VP16 (Figs. 3a and $5 \mathrm{a}, \mathrm{b})$. In summary, this shows that FoxH1dependent repression of $m i R-430$ is biologically relevant for the regulation of miR-430 target genes during maternal clearance.

\section{Discussion}

Here we describe a novel role for the transcription factor FoxH1 in negative regulation of $m i R-430$, and we provide evidence for a regulatory mechanism that is different from the previously described canonical role of FoxH1 in TGF-beta/Nodal signaling.

More than 20 years ago, FoxH1 was identified as the first Smad2-interacting transcription factor mediating transcriptional gene activation downstream of TGFbeta/Nodal signaling. Since then, FoxH1 has become established as a central transcriptional regulator of Nodal-induced mesendoderm induction and left-right patterning. Furthermore, a broad panel of genes were identified that are directly regulated by FoxH1/Smad2 via binding to proximal CAN binding sites [8-19]. Only recently, genome-wide ChiP analyses provided a more complex picture of highly dynamic FoxH1 chromatin occupancy in the early embryo. While these studies confirmed the importance of Nodal/Activin-induced CAN-interactions, they also revealed a much larger number of possibly Nodal/Activin independent NCDNA interactions of unknown function [12, 25]. Our analyses now provide first evidence for a functional relevance for $\mathrm{NC}$-interactions in gene regulation. Among other NC-targets, we identified the $m i R-430$ cluster as a novel in vivo target that is repressed by NC-FoxH1 interaction. Consistent with a Nodal independent function, we find that MZsur mutants display a stronger upregulation of $m i R-430$ as compared to Nodal signalingdeficient MZoep mutants (Fig. 2). Functional relevance for pri-miR-430 repression by FoxH1 was confirmed by showing that the increased pri-miR-430 levels in MZsur mutants correlate with a reduction of maternal transcripts that are targeted by miR-430 for de-adenylation and degradation. Our data suggest that the ubiquitous distribution of FoxH1 in the early embryo is required to restrict the level of miR-430 induction at the onset of zygotic gene expression. The patterned zygotic expression of FoxH1 during gastrulation and early somitogenesis [10] further implies that FoxH1 in addition to its role in mediating Nodal signaling might function in spatio-temporal controlled attenuation of miR-430dependent transcriptional silencing or mRNA clearance. Importantly, miR-430 is dampening not only maternal, but also a large number of zygotically expressed transcripts with various functions in early embryogenesis. Interestingly, this includes $l f t 2$ and $n d r 1$, which are both also directly regulated by CAN-FoxH1/Smad2-mediated gene activation [36, 38, 39]. Since both mechanisms for lft2 and ndr1 regulation, CAN-FoxH1-dependent feedback-activation and miR-430-mediated transcript 

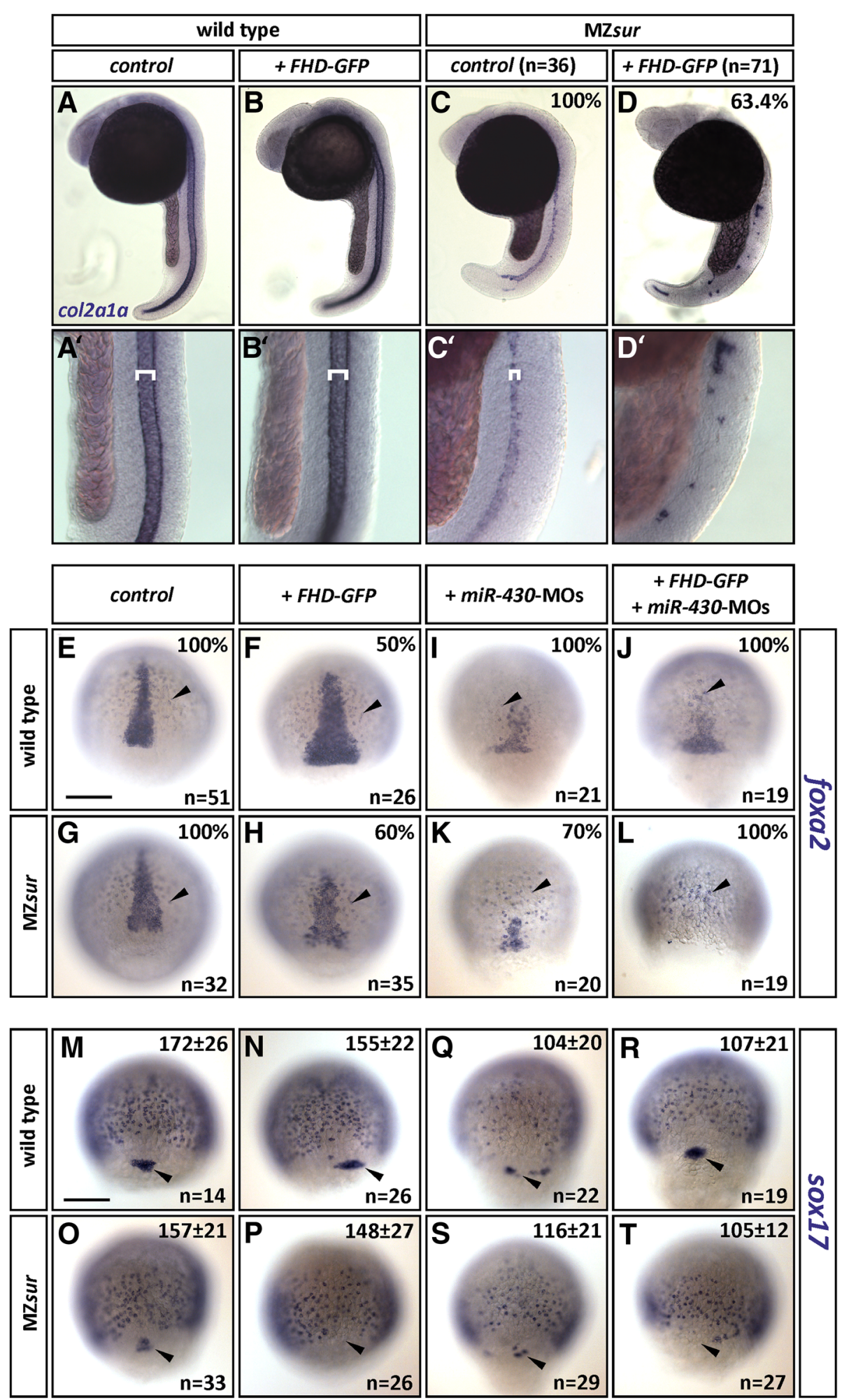

Fig. 4 (See legend on next page.) 
(See figure on previous page.)

Fig. 4 FHD-GFP interferes with severity of the MZsur mutant phenotype. a-d Wild-type (a, b) and MZsur mutant (c, d) embryos at 24hpf. col2ala in-situ staining in wild-type (un-injected control (a) or injected with FHD-GFP (b)) shows wild-type notochord of expected width (white brackets in enlarged sections $\mathbf{a}^{\prime}$ and $\mathbf{b}^{\prime}$ ). In uninjected MZsur embryos the width is reduced (c'). Injection of FHD-GFP in MZsur mutants enhances the phenotype (d/d'; note reduced size and additionally discontinuity of staining). e-I foxa2 in-situ hybridizations show reduction of axial mesoderm formation in MZsur mutants. Injection of FHD-GFP causes a broadened axial signal in 50\% of wild type embryos, but no reduction of axial cells (f), and strengthens the effect in $60 \%$ of the MZsur mutants (h). dre-miR-430 morpholinos (MOs) massively reduce axial foxa2 signals in both genotypes (l, k). Co-injection of FHD-GFP and MOs also results in a decreased staining (j, I) when compared to FHD-GFP injection (f, h). Percentage of embryos showing the same phenotype as in the image is given (upper right). $\mathbf{m}-\mathbf{t}$ sox 17 in-situ hybridizations show only slight reduction of endoderm after injection of MOs $(\mathbf{q}-\mathbf{t})$. Number of forerunner cells (black arrow) is reduced in MZsur control and after MOs injections (o, q, s). FHD-GFP lead to complete loss of forerunner cells (black arrow) in the majority of MZsur embryos ( $\boldsymbol{p}$, $\mathbf{t}$; see also Additional file 8: Statistical analysis of forerunner cells). Numbers of sox17-positive cells seen in dorsal view and standard deviation are given (upper right) as well as number of analyzed embryos ( $n$ ) (lower right). Size bars: $200 \mu \mathrm{m} . \gamma$ value was changed to 0.8 in each picture
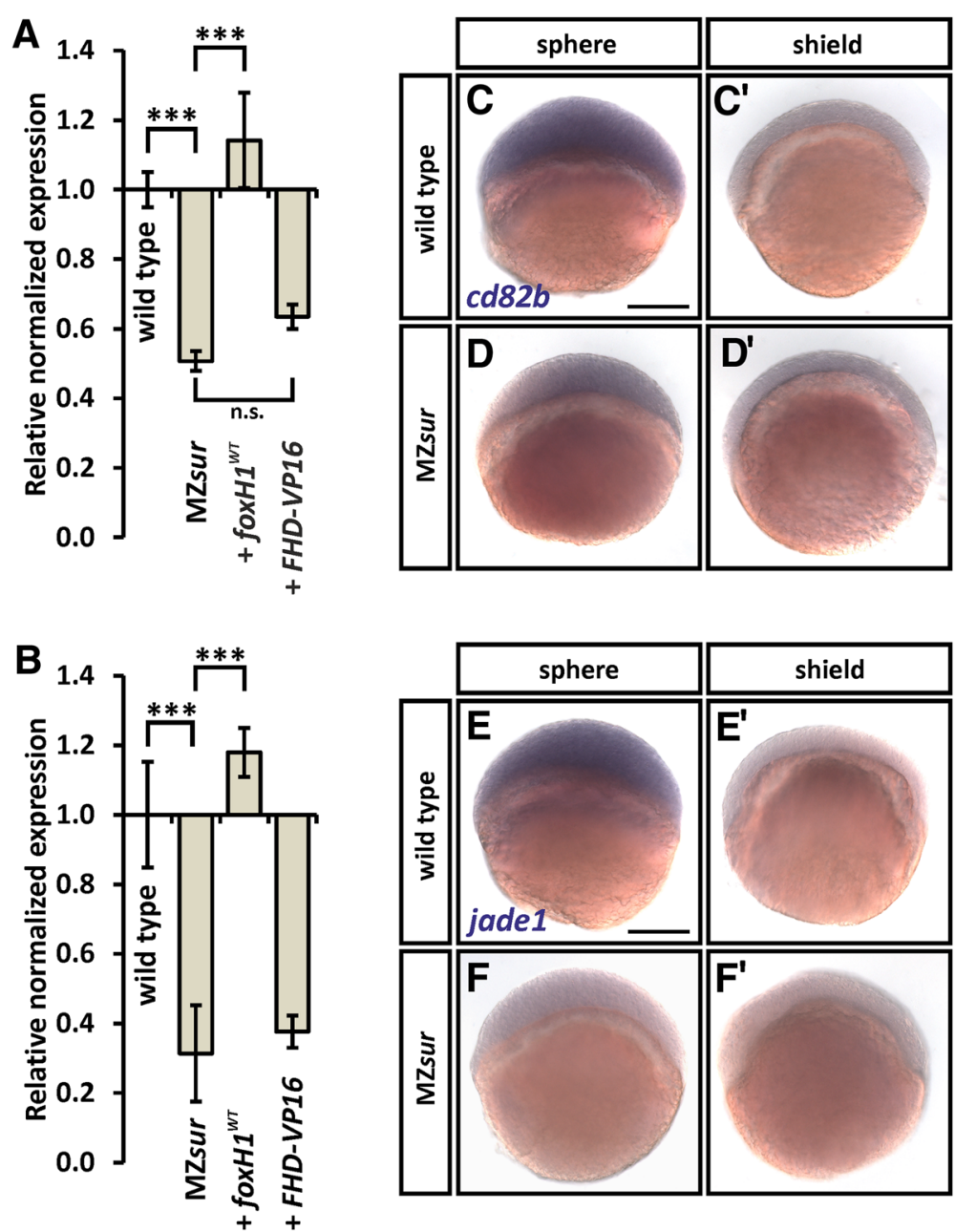

Fig. 5 FoxH1 blocks maternal clearance of $c d 82 b$ and jade1. a, b RT-qPCR analysis of cd82b (a) or jade1 (b) in embryos at 50\% epiboly with indicated genetic background. Massive reduction of expression is shown in MZsur mutants which cannot be rescued by injection of FHD-VP16 mRNA but is rescued via injection of foxH1 ${ }^{W T}$ mRNA. Error bars indicate standard error (SEM) from 2 biological replicates. Calculation of relative normalized expression, standard error, and significance was made with the Bio-Rad CFX Manager 3.1 software $\left.{ }^{* * *} p<0.001 ; n .5 . p \geq 0.05\right)$. For individual values, see also Additional file 5: Individual qPCR values. c-f WISH for cd82b and jade1 in wild type embryos (c, e) shows staining at sphere stage, but not at shield stage when miR-430 becomes active ( $\mathbf{c}^{\prime}$, $\mathbf{e}^{\prime}$ ). In MZsur mutants (d, $\mathbf{f}$ ), weak or no staining is visible for all stages indicating the negative role of FoxH1 in regulating miR-430 activity at early embryonic stages. Size bars $200 \mu m$ 
decay, are critical for balancing Nodal signaling, our data hint for a complex multilayered role of FoxH1 in directly and indirectly controlling and mediating Nodal activities in the early embryo. For future studies, it will be important to further characterize direct and indirect activities of FoxH1 on Nodal signaling and to study compensatory interactions between these activities.

\section{MZsur and MZmid embryo phenotypes may not reflect complete loss of FoxH1}

Our data suggest that the currently available foxH1 mutants sur and mid both can interfere with gene regulation and that some aspects of the MZsur and MZmid mutant phenotypes might not be seen in a truly null allele. In a previous study, it was shown that the stronger MZmid phenotype is rescued to an MZsur-like phenotype by an injection of foxH1 ${ }^{m 768}$ but not by foxH1 ${ }^{\text {mid }}$ RNA. Since injection of foxH1 $1^{\text {mid }}$ RNA in wild-type embryos caused no obvious defects, it was suggested that FoxH $1^{\mathrm{m} 768}$ retains residual activity that is missing in FoxH1 $1^{\text {mid }}$ [11]. Our results confirmed a generally normal morphology of wild-type embryos injected with the fox $H 1^{\text {mid }}$-like FHD-GFP mRNA, while they also revealed a broadened notochord in these embryos during gastrulation. Most relevant, we find that injection of FHD-GFP converted MZsur embryos into more severe MZmid-like embryos (Fig. 4). This notion is also consistent with the observed increase of miR-430 levels by FHD-GFP not only in wild type but also in MZsur mutants. Therefore, our data hint for a more complex mechanism in which FoxH1 $1^{m 768}$ may prevent or attenuate the stronger dominant-negative or possible neomorphic effects of FoxH $1^{\text {mid }}$. Interestingly, we also noticed that FHD-GFP has a stronger effect on miR-430 regulation as compared to FHD-VP16 and FHD-EN (Fig. 3a). Since the corresponding sur-mutant $F H D^{m 768}$-GFP is inactive, we consider FHD-GFP activities as specific to the FHD. Possibly, the robust activation and repression of CAN-FoxH1 targets by $F H D-V P 16$ and $F H D-E N$, respectively, is able to compensate for aspects seen in FHD-GFP injections.

While we focused on miR-430 regulation to determine functions of the different FoxH1 domains, our combined ChIP and expression data hint for a much broader role for NC-FoxH1 interactions in gene regulation. Consistent with this notion, we propose that the changed miR430 levels are not the major cause for the phenotypic differences between MZsur and MZmid. In case of a primary cause, the morpholino knock-down of miR-430 should have reduced mesoderm defects in FHD-GFP injected MZsur. Instead, a strong reduction or complete loss of axial mesoderm was observed in these embryos (Fig. 4i-l). Since miR-430s target hundreds of mRNAs, the morpholino injections might cause a dominant phenotype that overrides the expected axial mesoderm rescue by $m i R-430$ reduction. However, in wild-type embryos, injection of dre-miR-430 MOs was shown to reduce Nodal signaling by causing premature and increased translation of Lefty proteins ([36], see also Fig. 4l). While the complete loss of axial mesoderm in FHD-GFP and morpholino co-injected MZsur is consistent with a reduction of Nodal signaling by Lefty proteins, the strongly reduced lft1/2 mRNAs levels in MZsur mutants argue against such a mechanism [36]. In this context, the large number of NC-FoxH1 peaks suggest that FHD-GFP, similar to its effect on miR-430 expression, might interfere with other regulators of axial mesoderm formation. Consistent with this notion, our data suggest NC-interaction with Wnt, FGF, and retinoic acid signaling components (Fig. 4; Additional file 2: FoxH1-peaks and Annotation-50 $\mathrm{k}+20 \mathrm{k})$.

In conclusion, these studies show that for a detailed understanding of the early molecular events underlying mesendoderm formation, it is important to have a true null allele for FoxH1. In corresponding mutants, overexpression of distinct FoxH1 variants could be used to separate CAN- and $\mathrm{NC}-\mathrm{FoxH} 1$ functions and to determine the molecular factors responsible for the phenotypic differences between MZsur and MZmid [10, 11, 24].

\section{A new hypothetical model for FoxH1 regulation of miR- 430 locus}

The data generated in this study suggest that miR-430 repression by FoxH1 requires interaction of FoxH1 with at least two distinct genomic loci (Figs. 1 and 3). They also suggest that two major protein domains of FoxH1, the FHD and the SID, have specific functions in mediating these interactions and that this activity is independent of the central EH1 domain, which was shown to mediate Nodal-independent gene-repression via direct interaction with Groucho/TLE co-repressors (Fig. 3) [22, 23]. As SID$\mathrm{EN}$ is able to repress the miR-430 cluster independent of Nodal-signaling (demonstrated in MZoep mutants; Fig. 3d), our data suggest a SID interaction with chromatin that is independent of activated SMAD2.

Therefore, we propose a novel role of FoxH1 in connecting the miR-430 cluster with a distal regulatory element which then mediates repression per se, or another protein or protein complex does the work, as shown in our hypothetical model (Fig. 6a). Accordingly, we further propose that the currently available mutants dominantly interfere with chromatin scaffolding by association with contact sites either at the distal CAN-motif (Mid) or at the miR-430 cluster (Sur). At the onset of MZT, the inhibitory effect of FoxH1 is overcome by an unknown mechanism. Since Nanog, Pou5f1, and SoxB1 are known positive regulators of $m i R-430$ [47], FoxH1 and the changed chromatin scaffolding may prevent binding of these factors to miR-430 enhancer sites or block translation at 


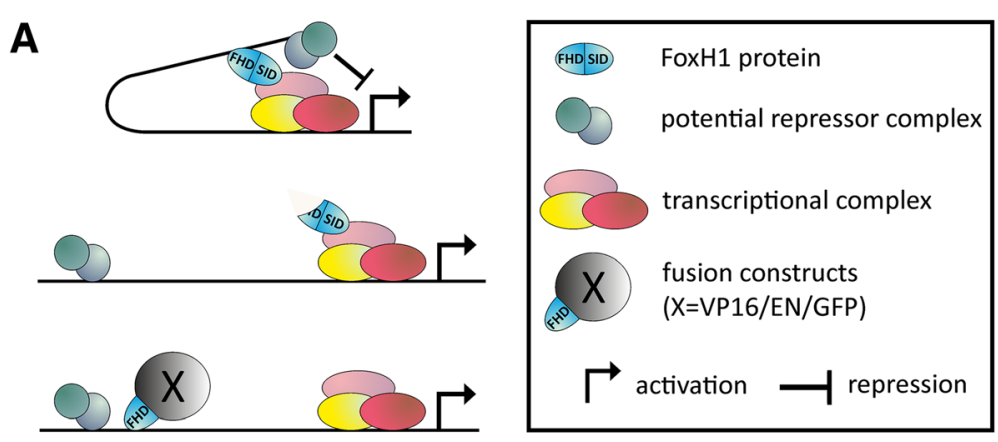

B

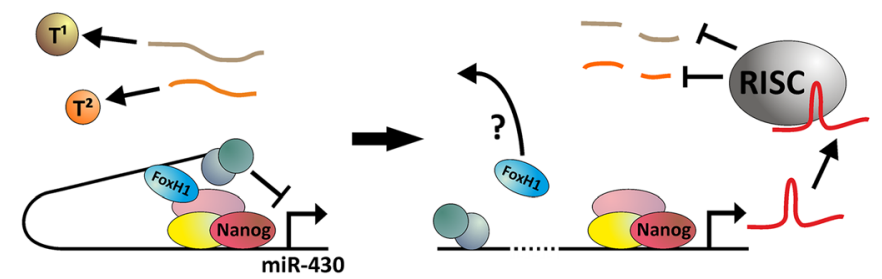

Fig. 6 A proposed model for the role of FoxH1 in the regulation of miR-430 activity. a Wild-type FoxH1 causes chromatin looping, preventing expression of miR-430. In MZsur mutants lacking the FHD, this loop is absent because FoxH1 binds only to the miR-430 cluster. If FoxH1 lacking a functional SID or FHD (due to mutation (m786) or replacement (VP16/GFP/EN)) is injected into wild types, higher expression of miR-430 occurs due to loss of looping and inhibitory regulation. b FoxH1 occupies non-canonical (NC) target sequences at miR-430 loci to induce chromatin scaffolding and prevent pri-miR-430 expression. With the onset of gastrulation, FoxH1 leaves the site, allowing miR-430 to become active and repress its targets $\left(T^{1} / T^{2}\right)$

these sites. It is not known whether Nanog/Pou5f1/ SoxB1 bind at miR-430 loci from the earliest developmental stages or can only occupy these binding sites once the FoxH1-initiated change in chromatin scaffolding disappears.

In the last decade, it has become clear that NC-DNA interactions of transcription factors are often associated with chromatin looping, bringing distantly located DNA domains closer together ([52], reviewed in [53-56]). Therefore, regulatory elements can influence distal genes not only on the same chromosome but also on different chromosomes [57]. Various examples in model organisms such as Drosophila and mice, as well as human cell culture systems, demonstrate high plasticity in the formation of the so-called Extremely Long-Range Promotor-Promotor Interactions (ELRIs), which are associated with the initiation and/or maintenance of gene activity, even during the earliest developmental stages ([57-62], reviewed in [63]). While the data we present are well explained by an involvement of FoxH1 in chromatin folding, further experiments are necessary to confirm this role. Several methods have been described for studying chromatin loops: $3 \mathrm{C}$ and derivatives, ChIA-PET, DNA fluorescent in situ hybridization (overview in [64-66]), and CRISPR/Cas9 guided in vivo chromatin labeling $[67,68]$. By using one or a combination of these methods, potential FoxH1-associated loops can be identified. Due to the complexity of the miR-430 loci, identifying relevant chromatin loops might not be as straightforward as for single and well-defined CAN-sites of a given transcriptional factor. However, our data also suggest that NCFoxH1 activities are not restricted to miR-430. Consistent with a possibly more global role of FoxH1 in early chromatin folding, we find that a large number NC-FoxH1 peaks are associated with genes that are either up- $(242 / 6154$ peaks) or downregulated (182/6154 peaks) in MZsur (Fig. 1c). In preliminary ChIP-qPCR studies that were set up to confirm selected NC-interactions of FoxH1, we noticed that enrichment of NC-peaks was variable in 4 and $5.5 \mathrm{hpf}$ samples while CAN-peaks were similarly enriched in these samples. In this context, the recently reported high number of dynamic FoxH1 chromatin interactions in early Xenopus development provides a hint for a possible conserved mechanism regulating dynamic interaction of FoxH1 at NC-sites [22, 23].

\section{Conclusions}

Overall, our data show that FoxH1, in addition to its established function downstream of TGF-beta/Nodalsignals [8-12], can regulate gene expression via indirect SID-mediated chromatin interaction. By identifying the microRNA-430 family members as functionally relevant targets for non-canonical FoxH1 regulation, our data place FoxH1 upstream of miR-430-regulated processes such as maternal clearance and balancing of Nodal 
signaling. In addition, the widespread distribution of NC binding sites of FoxH1, found not only in our study, but also in a recent ChIP analysis performed in Xenopus [22], hint for a more global role of FoxH1 in early embryonic chromatin folding. Therefore, this new knowledge of NC-FoxH1 functions provides us with important new insights into the molecular and epigenetic mechanisms underlying early zygotic gene regulation.

\section{Methods}

\section{Zebrafish lines}

The following lines were used: MZsur ${ }^{m 768 / m 768}$ [69], MZoep $^{\text {m134/m134 }}$ [70], and Tü (Tübingen) as wild-type control. Embryos were raised at $28.5^{\circ} \mathrm{C}$ and staged to hours post-fertilization or percentage of epiboly as described [71].

\section{mRNA injections}

foxH1 ${ }^{W T}$, eGFP-foxH1, FHD-VP16, FHD-GFP, FHD-EN (all described in [10]), $F H D^{\Delta \mathrm{EH} 1}-G F P$, fox $H 1^{m 768}$, foxH $1^{m 768}$-GFP, $5^{\prime}$ foxH1, and SID-EN were synthesized with the SP6 mMESSAGEmMACHINE in vitro transcription kit following the manufacturer's protocol (Life Technologies, AM1340). Embryos were injected at 1-2cell stage with $2 \mathrm{nl}(=90 \mathrm{pg})$ of each mRNA.

\section{Morpholino injections}

Morpholinos against dre-miR-430a, $b$, and $c$ were a kind gift from Caroline S. Hill [36]. Stock solutions of $2 \mathrm{mM}$ were diluted for injection at a 1:12 ratio in water. Two nanoliters of a mixture of the three MOs was injected in embryos at the 1-2-cell stage.

\section{Chromatin immunoprecipitation assay (ChIP)}

We used MZsur ${ }^{m 768 / m 768}$ embryos for the assay to exclude occupation of FoxH1 binding sites by endogenous FoxH1 proteins.

ChIP experiments were performed as described $[72,73]$ with minor changes. In total, 6000 foxH1 mutant embryos $\left(\mathrm{MZsur} r^{m 768 / m 768}\right)$ were injected each at 1-2-cell stage with $8 \mathrm{ng} / \mu \mathrm{l}$ ( $30 \mathrm{pg} / \mathrm{embryo})$ of $e G F P-f o x H 1 \mathrm{mRNA}$, encoding full-length FoxH1 with an N-terminal eGFP tag. Embryos were collected and cross-linked at $6 \mathrm{hpf}$. Chromatin was extracted and sheared into 100-200 bp fragments with the Bioruptor Sonicator (Diagenode, Belgium).

Anti-eGFP antibody (Torrey Pine Biolabs, 0715119, Protein A purified rabbit IgG) was pre-blocked and added (10 $\mu \mathrm{g} / 1000$ embryos) to the cross-linked samples and incubated overnight at $4{ }^{\circ} \mathrm{C}$. The next day, Dynabeads (Life Technologies, 10007D) were added to IP down protein-DNA complexes. Validated ChIP-DNA samples were amplified following the protocol of LinDa $[74,75]$. The concentration and fragment size of final amplification products were analyzed on a Bioanalyzer High Sensitivity DNA chip.

\section{High-throughput sequencing and data analysis}

LinDa-amplified ChIP-DNAs were sequenced on an Illumina HiSeq2000 at BGI (https://www.bgi.com/global/sequencing-services/epigenetics/chip-seq/) and produced a total of 24,932,016 raw clean $50 \mathrm{bp}$ single-end reads. These reads were then processed and filtered to remove low-quality reads and artifacts (e.g., residual LinDa adapters) resulting in 12,430,636 high-quality reads which were mapped to the zebrafish genome assembly $\mathrm{Zv9}$ / danRer7 using the BWA (v0.6.2) short read mapper [76].

\section{Peak calling}

Read alignments were first filtered using samtools to only retain uniquely mapping reads by removing all reads that map to multiple positions with equal alignment score. Then, peaks were called using MACS 1.4.2 [77] setting the $p$ values cutoff to $1 \mathrm{e}^{-4}$ and disallowing duplicate tags (reads) at the same position to avoid amplification biases.

This produced 23,724 raw peaks, of which 8446 had a $p$ value $<1 \mathrm{e}^{-5}$. These peaks were further filtered such that they did not overlap by more than $50 \%$ of their length with a non-UTR Ensembl exon and that they did not contain the following simple repeats: CTCTCTCT CTCTCTCTCTCTCTCTCTCT, AGAGAGAGA [GA] AGAGAGAGAGA, GAGAGAAA. This finally resulted in 16,908 peaks.

\section{FoxH1 motif de novo analysis}

The FoxH1 consensus binding motif was obtained by using meme-chip from the MEME suite [41] and the RSAT suite [42]. Sequences from peak regions that did not overlap with repeat-masked regions or genomic regions annotated as exons in the Ensembl database [78] but overlapped with conserved regions in fish and were within $5.5 \mathrm{~kb}$ of a TSS, were used as input sequences for motif prediction.

\section{Gene expression microarray}

Total RNA was extracted and purified from wild-type, MZsur and MZoep embryos using Qiagen RNeasy Mini Kit (74104). Three biological replicates were used for MZsur/MZoep and two for wild type, all of them showed RIN value $>8$ when analyzed with Agilent 2100 Bioanalyser. Agilent's Zebrafish Oligo Microarrays (V2), P/N G2519F (Agilent Microarray Design ID 019161) were hybridized according to the manufacturer's protocol. Raw data were analyzed using the Agi4x44PreProcessPackage ("R" package v1.22.0.) and the LIMMA-Package [79, 80]. Background correction mode was "half"; for normalization, the "quantile" option was used. The 
"Mean Signal" from the AFE software was used as signal, the "BGMedian Signal" as background. Data quality was confirmed using MA plots (data not shown).

\section{FoxH1 target gene annotations}

Genes were annotated as potential direct targets of FoxH1 by running our in-house developed peak annotation tool adapted from [81] and written in Perl. Briefly, the tool annotates ChIPseq peaks applying the following association rules: each RefSeq gene ( $\mathrm{Zv9} /$ danRer7) was assigned a basal regulatory domain $5 \mathrm{~kb}$ upstream and 1 $\mathrm{kb}$ downstream from the TSS. This basal domain could also overlap with basal domains from other genes. The basal domains were then extended in both directions to the nearest non-overlapping basal domain but no more than $50 \mathrm{~kb}$ upstream and $20 \mathrm{~kb}$ downstream from the TSS. Peaks within such regulatory domains (basal and extended) were associated with the corresponding genes. Genes associated with FoxH1 motif-containing peaks were defined as the "canonical FoxH1 regulated" genes.

\section{Analysis of FoxH1-SMADs direct targets}

The Smad2 SBRs were obtained from [44]. The original data based on $\mathrm{Zv} 7 /$ danRer5 was transposed to $\mathrm{Zv9} /$ danRer7. The transposed SBRs were then re-annotated and associated with genes. Association rules described above $(-5 \mathrm{~kb}-$ TSS $-+1 \mathrm{~kb}+$ extension $-50 \mathrm{~kb}-$ TSS $-+20 \mathrm{~kb})$ were applied. These SBRs were then centered at CAN-FoxH1 peaks, and their distribution was plotted in $250 \mathrm{bp}$ bins at the given distance to FoxH1 peaks. For building the random profiles, we generated the same number of random peaks as real peaks and analyzed them correspondingly.

\section{Genome-scale identification of potential FoxH1/SMAD targets}

The sequences around CAN-Foxh1 peaks were scanned with known SMAD consensus binding motifs derived from Genomatix matbase (www.genomatix.de) and SMADs/ FoxH1 pairs in which a SMAD motif (V\$Smad3.02, V\$Smad.01, V\$Smad3.01, and V\$Smad4.01) was located no more than $100 \mathrm{bp} 5$-prime of the FoxH1 motif were extracted and annotated.

\section{Mature miRNA extraction and RT-qPCR}

The total RNA was extracted and purified by miRNeasy Kit (Qiagen, 217184) from 30 embryos. cDNA synthesis and $\mathrm{qPCR}$ were performed following a previously published protocol [82]. The oligo 5'-GCAGGTCCAGTT TTTTTTTTTTTTTCTACCCC-3' was used for cDNA synthesis by Superscript III kit (Life Technologies, 18080051). The miR-103 was used for sample normalization in mature microRNA RT-qPCR. Primer data can be found in Additional file 9: qPCR primer.
RNA isolation and CDNA synthesis for pri-miRNA

TRIzol (Life Technologies, 15596026) was used to prepare total RNAs following the manufacturer's protocol. After DNA digestion by RNase-free DNaseI, total RNA was cleaned up by EtOH purification. One microgram RNA was used as template for reverse transcription using Maxima First Strand cDNA Synthesis Kit for RTqPCR (Thermo Scientific, \# K1641).

\section{Quantitative real-time PCR for pri-miRNA}

For each probe, $10 \mu \mathrm{l}$ of 1:10 diluted $\mathrm{cDNA}$ was mixed with $4 \mu \mathrm{l}$ HOT FIREPol Eva Green Mix (Solis Biodyne, 08-31-00001), $5.5 \mu \mathrm{l}$ water, and $0.5 \mu \mathrm{l}$ of one of the primer mixes in Additional file 9: qPCR primer $(100 \mathrm{pmol} / \mu \mathrm{l})$. Following protocol was used: initial step, $95^{\circ} \mathrm{C}$ for $15 \mathrm{~min}$; 40 cycles: $95^{\circ} \mathrm{C} 15 \mathrm{~s}, 60^{\circ} \mathrm{C} 30 \mathrm{~s}, 72^{\circ} \mathrm{C} 20 \mathrm{~s}$.

The data analysis is described elsewhere [83, 84]. If not indicated, differentially wild-type control was used for normalization. Significance was calculated using the program Bio-Rad CFX Manager 3.1 (Bio-Rad Laboratories, USA) (n. s. $p \geq 0.05$; $" p<0.05$; $* " p<0.01$; ${ }^{* * * *} p<$ 0.001). Microsoft Excel and CoralDraw were used for graphic composition.

\section{Whole mount in situ hybridization}

For whole-mount in-situ hybridization, cd82b-, jade1-, col2a1a-, foxa2-, and sox17-mRNA were used for generating digoxigenin-UTP (Roche Applied Science, 11175025910) labeled antisense probe. Staining followed a recently described protocol [85] using NBT/BCIP as dye substrate. For reducing variability, all wild-type or MZsur mutant embryos at different stages were stained in the same tube; probe and staining solutions were made for all and distributed to the single tubes.

For photo documentation, embryos were cleared in glycerol and imaged using a Leica CTR6000 microscope equipped with a Leica DFC 300FX camera (Leica Microsystems $\mathrm{GmbH}$; Wetzlar, Germany). CoralDraw was used for assembling figures. Single dorsal view images were used for quantification of sox17-positive cells.

\section{Additional files}

Additional file 1: Figure S1. miR-430 expression starts at midblastula transition. (PDF $76 \mathrm{~kb}$ )

Additional file 2: FoxH1-peaks and Annotation-50k+20k. (XLSX 1476 kb) Additional file 3: SBR to FoxH1 peaks. (XLSX $76 \mathrm{~kb}$ )

Additional file 4: Microarray vs. ChIP. (XLSX $778 \mathrm{~kb}$ )

Additional file 5: Individual qPCR values. (XLSX $90 \mathrm{~kb}$ )

Additional file 6: Overview of FoxH1 constructs. (PDF $241 \mathrm{~kb}$ )

Additional file 7: Figure S2. Regulation of CAN-target pitx2 and fgf3 by different constructs. (PDF $303 \mathrm{~kb}$ )

Additional file 8: Statistical analysis of forerunner cells. (XLSX $10 \mathrm{~kb}$ ) Additional file 9: $\mathrm{qPCR}$ primer. (XLSX $9 \mathrm{~kb}$ ) 


\section{Acknowledgements}

We thank Dzenana Tufegdzic for fish line maintenance and Sonja Töchterle for technical assistance. dre-miR-430 MOs were a kind gift from Caroline S. Hill (The Francis Crick Institute, London). Many thanks to Dominik Regele for help with some experiments and critical comment on the manuscript.

\section{Authors' contributions}

$\mathrm{DM}, \mathrm{PF}, \mathrm{HC}$, and FP conceived and designed the experiments. PF, HC, and FP performed the experiments. PF, HC, FP, DR, RK, and DM analyzed the data. PF and DM wrote the manuscript. All authors read and approved the final manuscript.

\section{Funding}

This research was supported by a grant from the FWF (FWF- P 20492-B09) to Dirk Meyer.

\section{Availability of data and materials}

All data generated or analyzed during this study are included in this published article, its additional files, and publicly available repositories. Following additional files are included:

Additional file 1. pdf: Figure S1. miR-430 expression starts at midblastula transition

Additional file 2. xlsx: FoxH1-peaks and Annotation-50k+20k

Additional file 3. xlsx: SBR to FoxH1 peaks

Additional file 4. xlsx: Microarray vs. ChIP

Additional file 5. xlsx: Individual gPCR values

Additional file 6. pdf: Overview of FoxH1 constructs

Additional file 7. pdf: Figure S2. Regulation of CAN-target pitx2 and fgf3 by different constructs.

Additional file 8. xlsx: Statistical analysis of forerunner cells

Additional file 9. xlsx: qPCR primer

Following datasets are available at public repository:

ChIP data on Gene Expression Omnibus (GSE133990;[40])

\section{Ethics approval and consent to participate}

Maintenance and handling of zebrafish followed the approved applications for animal experiments at the Bundesministerium für Wissenschaft, Forschung und Wirtschaft (BMWF) of the Republic of Austria: BMWF-66.008/ 0019-1I/3b/2013; BMWFW-66.008/0009-WF/II/3b/2014; BMWFW-66.008/0022WFN/3b/2016

\section{Consent for publication}

Not applicable.

\section{Competing interests}

The authors declare that they have no competing interests.

\section{Author details}

'Institute of Molecular Biology/CMBI, University of Innsbruck, Technikerstrasse 25, 6020 Innsbruck, Austria. ${ }^{2}$ Division of Bioinformatics, Biocenter, Innsbruck Medical University, Innrain 80, 6020 Innsbruck, Austria.

\section{Received: 15 April 2019 Accepted: 19 July 2019}

Published online: 30 July 2019

\section{References}

1. Mathieu J, Griffin K, Herbomel P, Dickmeis T, Strähle U, Kimelman D, et al. Nodal and Fgf pathways interact through a positive regulatory loop and synergize to maintain mesodermal cell populations. Development. 2004; 131(3):629-41.

2. Poulain M, Fürthauer $M$, Thisse $B$, Thisse $C$, Lepage $T$. Zebrafish endoderm formation is regulated by combinatorial Nodal, FGF and BMP signalling. Development. 2006;133(11):2189-200.

3. Bennett JT, Joubin K, Cheng S, Aanstad P, Herwig R, Clark M, et al. Nodal signaling activates differentiation genes during zebrafish gastrulation. Dev Biol. 2007:304(2):525-40.

4. Cordenonsi M, Dupont S, Maretto S, Insinga A, Imbriano C, Piccolo S. Links between tumor suppressors: p53 is required for TGF-beta gene responses by cooperating with Smads. Cell. 2003;113(3):301-14.

5. Ross S, Hill CS. How the Smads regulate transcription. Int J Biochem Cell Biol. 2008;40(3):383-408.
6. Schier AF. Nodal Morphogens. Cold Spring Harb Perspect Biol. 2009;1(5): a003459.

7. Kiecker C, Bates T, Bell E. Molecular specification of germ layers in vertebrate embryos. Cell Mol Life Sci. 2015;73:923-47.

8. Hoodless PA, Pye M, Chazaud C, Labbé E, Attisano L, Rossant J, et al. FoxH1 (Fast) functions to specify the anterior primitive streak in the mouse. Genes Dev. 2001;15(10):1257-71.

9. Kunwar PS, Zimmerman S, Bennett JT, Chen Y, Whitman M, Schier AF. Mixer/ Bon and FoxH1/Sur have overlapping and divergent roles in Nodal signaling and mesendoderm induction. Development. 2003;130(23):5589-99.

10. Pogoda HM, Solnica-Krezel L, Driever W, Meyer D. The zebrafish forkhead transcription factor FoxH1/Fast1 is a modulator of nodal signaling required for organizer formation. Curr Biol. 2000;10(17):1041-9.

11. Slagle CE, Aoki T, Burdine RD. Nodal-dependent mesendoderm specification requires the combinatorial activities of FoxH1 and eomesodermin. PLOS Genet. 2011;7(5):e1002072.

12. Nelson AC, Cutty SJ, Niini M, Stemple DL, Flicek P, Houart C, et al. Global identification of Smad2 and Eomesodermin targets in zebrafish identifies a conserved transcriptional network in mesendoderm and a novel role for Eomesodermin in repression of ectodermal gene expression. BMC Biol. 2014;12:81.

13. Saijoh Y, Adachi H, Sakuma R, Yeo CY, Yashiro K, Watanabe M, et al. Left-right asymmetric expression of lefty 2 and nodal is induced by a signaling pathway that includes the transcription factor FAST2. Mol Cell. 2000;5(1):35-47.

14. Yamamoto M, Meno C, Sakai Y, Shiratori H, Mochida K, Ikawa Y, et al. The transcription factor FoxH1 (FAST) mediates Nodal signaling during anteriorposterior patterning and node formation in the mouse. Genes Dev. 2001; 15(10):1242-56.

15. Kofron M, Puck H, Standley H, Wylie C, Old R, Whitman M, et al. New roles for FoxH1 in patterning the early embryo. Development. 2004;131(20):5065-78.

16. Silvestri C, Narimatsu M, von Both I, Liu Y, Tan NBJ, Izzi L, et al. Genomewide identification of Smad/Foxh1 targets reveals a role for Foxh1 in retinoic acid regulation and forebrain development. Dev Cell. 2008;14(3): $411-23$

17. Labbé E, Silvestri C, Hoodless PA, Wrana JL, Attisano L. Smad2 and Smad3 positively and negatively regulate TGF beta-dependent transcription through the forkhead DNA-binding protein FAST2. Mol Cell. 1998;2(1):109-20.

18. Zhou S, Zawel L, Lengauer C, Kinzler KW, Vogelstein B. Characterization of human FAST-1, a TGF beta and activin signal transducer. Mol Cell. 1998;2(1): $121-7$.

19. Kim SW, Yoon S-J, Chuong E, Oyolu C, Wills AE, Gupta R, et al. Chromatin and transcriptional signatures for Nodal signaling during endoderm formation in hESCs. Dev Biol. 2011;357(2):492-504.

20. Coda DM, Gaarenstroom T, East P, Patel H, Miller DSJ, Lobley A, et al. Distinct modes of SMAD2 chromatin binding and remodeling shape the transcriptional response to NODAL/Activin signaling. Elife. 2017;6: e22474

21. Beyer TA, Weiss A, Khomchuk Y, Huang K, Ogunjimi AA, Varelas $X$, et al. Switch enhancers interpret TGF- $\beta$ and Hippo signaling to control cell fate in human embryonic stem cells. Cell Rep. 2013;5(6):1611-24

22. Reid CD, Steiner AB, Yaklichkin S, Lu Q, Wang S, Hennessy M, et al. FoxH1 mediates a Grg4 and Smad2 dependent transcriptional switch in Nodal signaling during Xenopus mesoderm development. Dev Biol. 2016;414(1): $34-44$.

23. Charney RM, Forouzmand E, Cho JS, Cheung J, Paraiso KD, Yasuoka Y, et al. Foxh1 occupies cis-regulatory modules prior to dynamic transcription factor interactions controlling the mesendoderm gene program. Dev Cell. 2017; 40(6):595-607.e4.

24. Sirotkin HI, Gates MA, Kelly PD, Schier AF, Talbot WS. Fast1 is required for the development of dorsal axial structures in zebrafish. Curr Biol. 2000; 10(17):1051-4.

25. Nelson AC, Cutty SJ, Gasiunas SN, Deplae I, Stemple DL, Wardle FC. In vivo regulation of the zebrafish endoderm progenitor niche by T-box transcription factors. Cell Rep. 2017:19(13):2782-95.

26. Griffiths-Jones S. The microRNA registry. Nucleic Acids Res. 2004;32(Database issue):D109-11.

27. Bhattacharya M, Sharma AR, Sharma G, Patra BC, Nam J-S, Chakraborty $C$, et al. The crucial role and regulations of miRNAs in zebrafish development. Protoplasma. 2017;254(1):17-31.

28. Doench JG, Sharp PA. Specificity of microRNA target selection in translational repression. Genes Dev. 2004;18(5):504-11. 
29. Lewis BP, I-h S, Jones-Rhoades MW, Bartel DP, Burge CB. Prediction of mammalian microRNA targets. Cell. 2003;115(7):787-98.

30. Baskerville S, Bartel DP. Microarray profiling of microRNAs reveals frequent coexpression with neighboring miRNAs and host genes. RNA. 2005;11(3): $241-7$.

31. Nudelman G, Frasca A, Kent B, Sadler KC, Sealfon SC, Walsh MJ, et al. High resolution annotation of zebrafish transcriptome using long-read sequencing. Genome Res. 2018;28(9):1415-25.

32. Chen PY, Manninga H, Slanchev K, Chien M, Russo JJ, Ju J, et al. The developmental miRNA profiles of zebrafish as determined by small RNA cloning. Genes Dev. 2005;19(11):1288-93.

33. Giraldez AJ, Cinalli RM, Glasner ME, Enright AJ, Thomson JM, Baskerville S, et al. MicroRNAs regulate brain morphogenesis in zebrafish. Science. 2005; 308(5723):833-8.

34. Giraldez AJ, Mishima Y, Rihel J, Grocock RJ, van Dongen S, Inoue K, et al. Zebrafish MiR-430 promotes deadenylation and clearance of maternal mRNAs. Science. 2006:312(5770):75-9.

35. Mishima Y, Giraldez AJ, Takeda Y, Fujiwara T, Sakamoto H, Schier AF, et al. Differential regulation of germline mRNAs in soma and germ cells by zebrafish miR-430. Curr Biol. 2006;16(21):2135-42.

36. van Boxtel AL, Chesebro JE, Heliot C, Ramel M-C, Stone RK, Hill CS. A temporal window for signal activation dictates the dimensions of a nodal signaling domain. Dev Cell. 2015;35(2):175-85.

37. Svoboda P, Flemr M. The role of miRNAs and endogenous siRNAs in maternal-to-zygotic reprogramming and the establishment of pluripotency. EMBO Rep. 2010;11(8):590-7.

38. Choi W-Y, Giraldez AJ, Schier AF. Target protectors reveal dampening and balancing of Nodal agonist and antagonist by miR-430. Science. 2007; 318(5848):271-4

39. Rosa A, Spagnoli FM, Brivanlou AH. The miR-430/427/302 family controls mesendodermal fate specification via species-specific target selection. Dev Cell. 2009;16(4):517-27.

40. Fischer P, Rieder D, Meyer D. Genome-wide maps of binding sites of FoxH1 in 6hpf epiboly-stage zebrafish embryos.: Gene Expression Omnibus; 2019. Available from: URL: https:/www.ncbi.nlm.nih.gov/geo/query/acc.cgi?acc=GSE133990.

41. Machanick P, Bailey TL. MEME-ChIP: motif analysis of large DNA datasets. Bioinformatics. 2011;27(12):1696-7.

42. Thomas-Chollier M, Defrance M, Medina-Rivera A, Sand O, Herrmann C, Thieffry D, et al. RSAT 2011: regulatory sequence analysis tools. Nucleic Acids Res. 2011;39(Web Server issue):W86-91.

43. Thomas-Chollier M, Darbo E, Herrmann C, Defrance M, Thieffry D, van Helden J. A complete workflow for the analysis of full-size ChIP-seq (and similar) data sets using peak-motifs. Nat Protoc. 2012;7(8):1551-68.

44. Liu Z, Lin X, Cai Z, Zhang Z, Han C, Jia S, et al. Global identification of SMAD2 target genes reveals a role for multiple co-regulatory factors in zebrafish early gastrulas*. J Biol Chem. 2011;286(32):28520-32.

45. UCSC Genome Browser. UCSC Genome Browser on Zebrafish Jul. 2010 (Zv9/ danRer7) Assembly; 2019. Available from: URL: http://genome.ucscedu/cgi-bin/ hgTracks?db=danRer7\&lastVirtModeType=default\&lastVirtModeExtraState= \&virtModeType $=$ default\&virtMode $=0 \&$ nonVirtPosition $=\&$ position $=$ chr $4 \% 3 \mathrm{~A} 2$ 7996539\%2D28023201\&hgsid=738451989_|AsST8JDtzh4uRXug8v5OS3tkVuR.

46. Pézeron G, Lambert G, Dickmeis T, Strähle U, Rosa FM, Mourrain P. Rasl11 b knock down in zebrafish suppresses one-eyed-pinhead mutant phenotype. PLoS One. 2008;3(1):e1434.

47. Lee MT, Bonneau AR, Takacs CM, Bazzini AA, DiVito KR, Fleming ES, et al. Nanog, Pou5f1 and SoxB1 activate zygotic gene expression during the maternal-to-zygotic transition. Nature. 2013:503(7476):360-4.

48. Dong JT, Lamb PW, Rinker-Schaeffer CW, Vukanovic J, Ichikawa T, Isaacs JT, et al. KAl1, a metastasis suppressor gene for prostate cancer on human chromosome 11p11.2. Science. 1995;268(5212):884-6.

49. Briese J, Schulte HM, Sajin M, Bamberger C, Redlin K, Milde-Langosch K, et al. Correlations between reduced expression of the metastasis suppressor gene KAl- 1 and accumulation of p53 in uterine carcinomas and sarcomas, Virchows Arch. 2008;453(1):89-96

50. Chitalia VC, Foy RL, Bachschmid MM, Zeng L, Panchenko MV, Zhou MI, et al. Jade-1 inhibits Wnt signaling by ubiquitinating $\beta$-catenin and mediates Wnt pathway inhibition by pVHL. Nat Cell Biol. 2008;10(10):1208-16.

51. Borgal L, Habbig S, Hatzold J, Liebau MC, Dafinger C, Sacarea I, et al. The ciliary protein nephrocystin-4 translocates the canonical Wnt regulator Jade-1 to the nucleus to negatively regulate $\beta$-catenin signaling. J Biol Chem. 2012;287(30):25370-80.
52. Ghavi-Helm Y, Klein FA, Pakozdi T, Ciglar L, Noordermeer D, Huber W, et al. Enhancer loops appear stable during development and are associated with paused polymerase. Nature. 2014;512(7512):96-100.

53. Sandhu KS, Li G, Poh HM, Quek YLK, Sia YY, Peh SQ, et al. Large-scale functional organization of long-range chromatin interaction networks. Cell Rep. 2012;2(5):1207-19.

54. de Graaf CA, van Steensel B. Chromatin organization: form to function. Curr Opin Genet Dev. 2013;23(2):185-90.

55. Slattery M, Zhou T, Yang L, Machado ACD, Gordân R, Rohs R. Absence of a simple code: how transcription factors read the genome. Trends Biochem Sci. 2014;39(9):381-99.

56. Levine M, Cattoglio C, Tjian R. Looping back to leap forward: transcription enters a new era. Cell. 2014;157(1):13-25.

57. Joshi O, Wang S-Y, Kuznetsova T, Atlasi Y, Peng T, Fabre PJ, et al. Dynamic reorganization of extremely long-range promoter-promoter interactions between two states of pluripotency. Cell Stem Cell. 2015; 17(6):748-57.

58. Amano T, Sagai T, Tanabe H, Mizushina Y, Nakazawa H, Shiroishi T. Chromosomal dynamics at the Shh locus: limb bud-specific differential regulation of competence and active transcription. Dev Cell. 2009;16(1):47-57.

59. Sanyal A, Lajoie B, Jain G, Dekker J. The long-range interaction landscape of gene promoters. Nature. 2012;489(7414):109-13.

60. Bonn S, Zinzen RP, Girardot C, Gustafson EH, Perez-Gonzalez A, Delhomme $\mathrm{N}$, et al. Tissue-specific analysis of chromatin state identifies temporal signatures of enhancer activity during embryonic development. Nat Genet. 2012;44(2):148-56

61. Hensel Z, Weng X, Lagda AC, Xiao J. Transcription-factor-mediated DNA looping probed by high-resolution, single-molecule imaging in live $E$. coli cells. PLoS Biol. 2013;11(6):e1001591.

62. Dixon JR, Jung I, Selvaraj S, Shen Y, Antosiewicz-Bourget JE, Lee AY, et al. Chromatin architecture reorganization during stem cell differentiation. Nature. 2015:518(7539):331-6.

63. Cournac A, Plumbridge J. DNA looping in prokaryotes: experimental and theoretical approaches. J Bacteriol. 2013;195(6):1109-19.

64. Fullwood MJ, Ruan Y. ChIP-based methods for the identification of longrange chromatin interactions. J Cell Biochem. 2009:107(1):30-9.

65. de Wit $\mathrm{E}$, de Laat W. A decade of $3 \mathrm{C}$ technologies: insights into nuclear organization. Genes Dev. 2012;26(1):11-24.

66. Fraser J, Williamson I, Bickmore WA, Dostie J. An overview of genome organization and how we got there: from FISH to Hi-C. Microbiol Mol Biol Rev. 2015;79(3):347-72

67. Ma H, Naseri A, Reyes-Gutierrez P, Wolfe SA, Zhang S, Pederson T. Multicolor CRISPR labeling of chromosomal loci in human cells. Proc Natl Acad Sci U S A. 2015;112(10):3002-7.

68. Deng W, Shi X, Tjian R, Lionnet T, Singer RH. CASFISH: CRISPR/Cas9mediated in situ labeling of genomic loci in fixed cells. Proc Natl Acad Sci U S A. 2015:112(38):11870-5.

69. Brand M, Heisenberg CP, Warga RM, Pelegri F, Karlstrom RO, Beuchle D, et al. Mutations affecting development of the midline and general body shape during zebrafish embryogenesis. Development. 1996;123:129-42.

70. Schier AF, Neuhauss SC, Harvey M, Malicki J, Solnica-Krezel L, Stainier DY, et al. Mutations affecting the development of the embryonic zebrafish brain. Development. 1996:123:165-78.

71. Kimmel CB, Ballard WW, Kimmel SR, Ullmann B, Schilling TF. Stages of embryonic development of the zebrafish. Dev Dyn. 1995:203(3):253-310.

72. Wardle FC, Odom DT, Bell GW, Yuan B, Danford TW, Wiellette EL, et al. Zebrafish promoter microarrays identify actively transcribed embryonic genes. Genome Biol. 2006;7(8):R71.

73. Morley RH, Lachani K, Keefe D, Gilchrist MJ, Flicek P, Smith JC, et al. A gene regulatory network directed by zebrafish No tail accounts for its roles in mesoderm formation. Proc Natl Acad Sci U S A. 2009;106(10):3829-34.

74. Shankaranarayanan P, Mendoza-Parra M-A, Walia M, Wang L, Li N, Trindade LM, et al. Single-tube linear DNA amplification (LinDA) for robust ChIP-seq. Nat Methods. 2011;8(7):565-7.

75. Shankaranarayanan P, Mendoza-Parra M-A, van Gool W, Trindade LM, Gronemeyer $\mathrm{H}$. Single-tube linear DNA amplification for genome-wide studies using a few thousand cells. Nat Protoc. 2012;7(2):328-38.

76. Li H, Durbin R. Fast and accurate long-read alignment with BurrowsWheeler transform. Bioinformatics. 2010;26(5):589-95.

77. Zhang Y, Liu T, Meyer CA, Eeckhoute J, Johnson DS, Bernstein BE, et al. Model-based analysis of ChIP-Seq (MACS). Genome Biol. 2008;9(9):R137. 
78. Flicek P, Amode MR, Barrell D, Beal K, Billis K, Brent S, et al. Ensembl 2014. Nucleic Acids Res. 2014;42(Database issue):D749-55.

79. Wettenhall JM, Smyth GK. limmaGUI: a graphical user interface for linear modeling of microarray data. Bioinformatics. 2004;20(18):3705-6.

80. Smyth GK. limma: Linear Models for Microarray Data. In: Gentleman R, Carey VJ, Huber W, Irizarry RA, Dudoit S, editors. Bioinformatics and computational biology solutions using R and Bioconductor. New York: Springer-Verlag; 2005. p. 397-420. (Statistics for Biology and Health).

81. Hiller M, Agarwal S, Notwell JH, Parikh R, Guturu H, Wenger AM, et al, Computational methods to detect conserved non-genic elements in phylogenetically isolated genomes: application to zebrafish. Nucleic Acids Res. 2013;41(15):e151.

82. Balcells I, Cirera S, Busk PK. Specific and sensitive quantitative RT-PCR of miRNAs with DNA primers. BMC Biotechnol. 2011;11:70.

83. Livak KJ, Schmittgen TD. Analysis of relative gene expression data using real-time quantitative PCR and the 2(-Delta Delta C(T)) method. Methods. 2001;25(4):402-8.

84. Cumming G, Fidler F, Vaux DL. Error bars in experimental biology. J Cell Biol. 2007;177(1):7-11.

85. Hauptmann G, Gerster T. Multicolor whole-mount in situ hybridization. Methods Mol Biol. 2000;137:139-48.

\section{Publisher's Note}

Springer Nature remains neutral with regard to jurisdictional claims in published maps and institutional affiliations.

Ready to submit your research? Choose BMC and benefit from:

- fast, convenient online submission

- thorough peer review by experienced researchers in your field

- rapid publication on acceptance

- support for research data, including large and complex data types

- gold Open Access which fosters wider collaboration and increased citations

- maximum visibility for your research: over $100 \mathrm{M}$ website views per year

At $\mathrm{BMC}$, research is always in progress.

Learn more biomedcentral.com/submissions 